Pacific Journal of Mathematics

STRONGLY ANALYTIC SUBSPACES AND STRONGLY 


\title{
STRONGLY ANALYTIC SUBSPACES AND STRONGLY DECOMPOSABLE OPERATORS
}

\author{
JON C. SNADER
}

\begin{abstract}
Strongly analytic subspaces, in the sense of Lange, are studied, and a characterization of them in terms of Bishop's condition $(\beta)$ is given. These results are used to obtain a characterization of strongly decomposable operators on a reflexive Banach space in terms of strongly analytic subspaces.
\end{abstract}

1. Introduction. In [10], Lange introduced the notion of strongly analytic subspaces, and investigated some of their properties. In this paper, we continue that investigation, and apply our results to the study of strongly decomposable operators. In $\$ 2$, we study strongly analytic subspaces; here, the main result is Theorem 2.1 which characterizes strongly analytic subspaces in terms of a certain analytic condition due to Bishop. This result is then used to identify various sufficient conditions for a subspace to be strongly analytic, and to obtain certain stability results. In $\S 3$, Theorem 2.1 is combined with a result of Lange to obtain a characterization of strongly decomposable operators on a reflexive Banach space in terms of strongly analytic subspaces.

Since the leitmotif in this paper is the interplay between strongly analytic subspaces and Bishop's condition, we begin with their definition.

1.1. Definition [10]. Let $T$ be a bounded linear operator on a Banach space $X$. A $T$-invariant subspace $Y$ is said to be strongly analytic for $T$ if whenever $f_{n}: V \rightarrow X, n=1,2, \ldots$, is a sequence of $X$-valued analytic functions defined on an open subset $V$ of the complex plane such that $\operatorname{dist}\left((\lambda-T) f_{n}(\lambda), Y\right) \rightarrow 0$ uniformly on compact subsets of $V$, it follows that $\operatorname{dist}\left(f_{n}(\lambda), Y\right) \rightarrow 0$ uniformly on compact subsets of $V$.

1.2. Definition [2]. A bounded linear operator $T$ on a Banach space $X$ is said to possess Bishop's condition $(\beta)$, or condition $(\beta)$ if whenever $f_{n}$ : $V \rightarrow X, n=1,2, \ldots$, is a sequence of $X$-valued analytic functions defined on an open set $V$ of the complex plane such that $(\lambda-T) f_{n}(\lambda) \rightarrow 0$ uniformly (in norm) on compact subsets of $V$, it follows that $f_{n}(\lambda) \rightarrow 0$ uniformly (in norm) on compact subsets of $V$. 
1.3. Definition [7]. Let $T$ be a bounded linear operator. A $T$-invariant subspace $Y$ is said to be spectral maximal for $T$ if for any $T$-invariant subspace $Z$ such that $\sigma(T \mid Z) \subset \sigma(T \mid Y)$ we have that $Z \subset Y$.

1.4. Definition [7]. A bounded linear operator $T$ on a Banach space $X$ is said to be a decomposable operator if for every finite system $\left\{G_{1}, \ldots, G_{n}\right\}$ of open subsets of $C$ that cover $\sigma(T)$, there exist spectral maximal subspaces $Y_{1}, \ldots, Y_{n}$ such that

(1) $X=Y_{1}+\cdots+Y_{n}$,

(2) $\sigma\left(T \mid Y_{j}\right) \subset G_{j}$ for $j=1, \ldots, n$.

The following remarkable characterization is due to Lange [9].

1.5. THEOREM (Lange's Theorem). A bounded linear operator $T$ on a reflexive Banach space is decomposable if and only if $T$ and its adjoint $T^{*}$ enjoy condition $(\beta)$.

The next lemma is a compilation of some results we will need concerning condition $(\beta)$. The straightforward proofs can be found in $[11]$.

1.6. LEMMA. Let $T$ be a bounded linear operator on a Banach space.

(1) If $\sigma(T)$ is totally disconnected, then Thas condition $(\beta)$.

(2) If $Y$ is a T-invariant subspace, and Thas condition $(\beta)$, then so does the restriction operator $T \mid Y$.

(3) If the operator $S$ is (topologically) linearly isomorphic to $T$, then $S$ has condition $(\beta)$ if and only if $T$ does.

(4) If $T=T_{1} \oplus T_{2}: X_{1} \oplus X_{2} \rightarrow X_{1} \oplus X_{2}$, then $T$ has condition ( $\beta$ ) if and only if $T_{j}$ does for $j=1,2$.

2. Strongly analytic subspaces. Since an operator $T$ has condition ( $\beta$ ) if and only if the zero subspace is $T$-strongly analytic, it is clear that the two concepts are closely related. The following characterization makes this relationship precise.

2.1. THEOREM. If $Y$ is a T-invariant subspace, then $Y$ is $T$-strongly analytic if and only if the quotient operator $T^{Y}$ induced by $Y$ on $X / Y$ has condition $(\beta)$.

Proof. Suppose that $T^{Y}$ has condition $(\beta)$, that $f_{n}: V \rightarrow X, n=$ $1,2, \ldots$, are $X$-valued analytic functions, that $\operatorname{dist}\left((\lambda-T) f_{n}(\lambda), Y\right) \rightarrow 0$ 
uniformly on compact subsets of $V$ and that $K \subset V$ is compact. Then we have that $\left(\lambda-T^{Y}\right)\left[f_{n}(\lambda)\right] \rightarrow 0$ uniformly for $\lambda$ in $K$, where for $x$ in $X$, $[x]$ is the coset in $X / Y$ containing $x$. Since $T^{Y}$ has condition $(\beta)$, we conclude that $\left[f_{n}(\lambda)\right] \rightarrow 0$ uniformly for $\lambda$ in $K$, and this is equivalent to $\operatorname{dist}\left(f_{n}(\lambda), Y\right) \rightarrow 0$ uniformly for $\lambda$ in $K$. Thus $Y$ is strongly analytic for $T$.

For the reverse implication, suppose that $Y$ is $T$-strongly analytic, that $f_{n}: V \rightarrow X / Y, n=1,2, \ldots$, are $(X / Y)$-valued analytic functions, and that $\left(\lambda-T^{Y}\right) f_{n}(\lambda) \rightarrow 0$ uniformly on compact subsets of $V$. Let $K \subset V$ be compact. By a standard compactness argument, we may suppose that $K$ is a closed disk centered at $\lambda_{0}$, and that there exists another disk $U$ centered at $\lambda_{0}$ such that $K \subset U \subset \bar{U} \subset V$. If $f_{n}(\lambda)=\sum_{j=0}^{\infty}\left[x_{j}\right]\left(\lambda-\lambda_{0}\right)^{j}$ is the series expansion of $f_{n}$ for $\lambda$ in $U$, we can choose $x_{j}$ in $\left[x_{j}\right]$ such that the series $F_{n}(\lambda):=\sum_{j=0}^{\infty} x_{j}\left(\lambda-\lambda_{0}\right)^{J}$ converges uniformly for $\lambda$ in $U$. Note that $F_{n}$ is an $X$-valued analytic function such that $\left[F_{n}(\lambda)\right]=f_{n}(\lambda)$ for $\lambda$ in $U$. Thus we have $\left(\lambda-T^{Y}\right)\left[F_{n}(\lambda)\right] \rightarrow 0$ uniformly for $\lambda$ in $K$, so that $\left[(\lambda-T) F_{n}(\lambda)\right] \rightarrow 0$ uniformly for $\lambda$ in $K$, which implies that $\operatorname{dist}\left((\lambda-T) F_{n}(\lambda), Y\right) \rightarrow 0$ uniformly for $\lambda$ in $K$. Since $Y$ is strongly analytic for $T$ by hypothesis, we conclude that $\operatorname{dist}\left(F_{n}(\lambda), Y\right) \rightarrow 0$ uniformly for $\lambda$ in $K$, and therefore that $f_{n}(\lambda)=\left[F_{n}(\lambda)\right] \rightarrow 0$ uniformly for $\lambda$ in $K$. Thus $T^{Y}$ has condition $(\beta)$, and the proof is complete.

The next theorem generalizes Corollaries 9 and 10 of [10].

2.2. THEOREM. If a bounded linear operator has totally disconnected spectrum, then every $T$-invariant subspace $Y$ is $T$-strongly analytic.

Proof. Since $\sigma(T)$ is totally disconnected, $\sigma(T \mid Y) \subset \sigma(T)$. This last inclusion follows from the facts that $\sigma(T \mid Y)$ can grow only by filling in the "holes" in the components of $\sigma(T)$, and that the components of $\sigma(T)$ are points. Thus $\sigma\left(T^{Y}\right) \subset \sigma(T \mid Y) \cup \sigma(T)=\sigma(T)$. By Lemma 1.6, $T^{Y}$ has condition $(\beta)$, so that $Y$ is $T$-strongly analytic by Theorem 2.1 .

2.3. THEOREM. Let $T_{1} \oplus T_{2}$ be a bounded linear operator on a Banach space $X_{1} \oplus X_{2}$. Then the subspace $Y_{1} \oplus Y_{2}$ is strongly analytic for $T_{1} \oplus T_{2}$ if and only if $Y_{j}$ is strongly analytic for $T_{j}, j=1,2$.

Proof. By Theorem 2.1, $Y_{1} \oplus Y_{2}$ is strongly analytic for $T_{1} \oplus T_{2}$ if and only if $\left(T_{1} \oplus T_{2}\right)^{Y_{1} \oplus Y_{2}}$ has condition $(\beta)$, and by Lemma 1.6, this last condition obtains if and only if $T_{j}^{Y_{j}}$ has condition $(\beta)$ for $j=1,2$. Another application of Theorem 2.1 completes the proof. 
The next characterization improves on a result of Lange.

2.4. THEOREM. Let $T$ be a bounded linear operator on a Banach space $X$, and let $E$ be a bounded projection on $X$ that commutes with $T$. Then $E X$ is a $T$-strongly analytic subspace if and only if $T \mid(I-E) X$ has condition $(\beta)$.

Proof. Let $Y:=E X$ and $Z:=(I-E) X$, so that $X=Y \oplus Z$. Then $T^{Y}$ is linearly isomorphic to $T \mid Z$; hence by Lemma 1.6, $T^{Y}$ has condition ( $\beta$ ) if and only if $T \mid Z$ does. Thus by Theorem $2.1, Y$ is $T$-strongly analytic if and only if $T \mid Z$ has condition $(\beta)$.

2.5. Corollary. Let $T$ and $E$ be as above, and suppose, in addition, that $T$ enjoys condition $(\beta)$. Then $E X$ is T-strongly analytic.

Proof. This follows immediately from the theorem and Lemma 1.6.

2.6. Corollary [10]. Let $T$ be decomposable, and let $E$ be a commuting projection. Then $E X$ is $T$-strongly analytic.

Proof. By [6], a decomposable operator has condition $(\beta)$.

2.7. THEOREM. If a bounded linear operator Thas condition $(\beta)$, then its kernel, $\operatorname{ker} T$, is strongly analytic for $T$.

Proof. Let $M:=\operatorname{ker} T, f_{n}: V \rightarrow X, n=1,2, \ldots$, be $X$-valued analytic functions, and suppose that $\operatorname{dist}\left((\lambda-T) f_{n}(\lambda), M\right) \rightarrow 0$ uniformly on compact subsets of $V$. Let $K \subset V$ be compact. We first observe that it suffices to suppose that $\lambda=0$ is not in $K$. To see this, note that by a standard compactness argument we may assume that $K$ is a disk whose boundary does not contain 0 . Now if $\operatorname{dist}\left(f_{n}(\lambda), M\right) \rightarrow 0$ uniformly on compact sets not containing 0 , it will do so on the boundary of $K$, and hence, by the Maximum principle, on all of $K$.

Now $\operatorname{dist}\left((\lambda-T) T f_{n}(\lambda), T M\right) \rightarrow 0$ uniformly for $\lambda$ in $K$, and since $T M=(0)$, we conclude that $(\lambda-T) T f_{n}(\lambda) \rightarrow 0$ uniformly for $\lambda$ in $K$. But $T$ has condition $(\beta)$, so $T f_{n}(\lambda) \rightarrow 0$ uniformly for $\lambda$ in $K$.

Therefore, for $\lambda$ in $K$, we have that

$$
\operatorname{dist}\left(\lambda f_{n}(\lambda), M\right) \leq \operatorname{dist}\left((\lambda-T) f_{n}(\lambda), M\right)+\left\|T f_{n}(\lambda)\right\| \rightarrow 0
$$

uniformly, and so $\operatorname{dist}\left(f_{n}(\lambda), M\right) \rightarrow 0$ uniformly for $\lambda$ in $K$ since, by assumption, $\lambda=0$ is not in $K$. 
The next theorem is a slight generalization of the last one.

2.8. TheOREM. Suppose that $Y$ is a T-strongly analytic subspace, and let $M=\operatorname{ker} T$. Then $\overline{Y+M}$ is T-strongly analytic.

Proof. Suppose that $f_{n}: V \rightarrow X, n=1,2, \ldots$, are $X$-valued analytic functions and that $\operatorname{dist}\left((\lambda-T) f_{n}(\lambda), \overline{Y+M}\right) \rightarrow 0$ uniformly on compact subsets of $V$. Let $K \subset V$ be compact. Now $T(\overline{Y+M}) \subset Y$, so that $\operatorname{dist}\left((\lambda-T) T f_{n}(\lambda), Y\right) \rightarrow 0$ uniformly for $\lambda$ in $K$. Thus, by hypothesis, $\operatorname{dist}\left(T f_{n}(\lambda), Y\right) \rightarrow 0$ uniformly for $\lambda$ in $K$. Let $\varepsilon>0$ be given; for every $\lambda$ in $K$, and every positive integer $n$, there exist $y_{\lambda, n}$ in $\overline{Y+M}$ and $y_{\lambda, n}^{\prime}$ in $Y$, and there exists a positive integer $N$ such that if $n>N$, then

$$
\left\|(\lambda-T) f_{n}(\lambda)-y_{n, \lambda}\right\|<\varepsilon / 2 \text { and }\left\|T f_{n}(\lambda)-y_{\lambda, n}^{\prime}\right\|<\varepsilon / 2 .
$$

Now for $n>N$, and $\lambda$ in $K$, we have

$$
\operatorname{dist}\left(\lambda f_{n}(\lambda), \overline{Y+M}\right) \leq\left\|(\lambda-T) f_{n}(\lambda)-y_{\lambda, n}\right\|+\left\|T f_{n}(\lambda)-y_{\lambda, n}^{\prime}\right\|<\varepsilon .
$$

Thus $\operatorname{dist}\left(f_{n}(\lambda), \overline{Y+M}\right) \rightarrow 0$ uniformly for $\lambda$ in $K$, where, as in Theorem 2.7, we may assume that $\lambda=0$ is not in $K$.

We also have a result analogous to Theorem 2.7 , but for the range of the operator.

2.9. THEOREM. Let $Y$ be a T-strongly analytic subspace. Then $\overline{T Y}$ is also T-strongly analytic.

Proof. Let $f_{n}: V \rightarrow X, n=1,2, \ldots$, be $X$-valued analytic functions, and let $\operatorname{dist}\left((\lambda-T) f_{n}(\lambda), \overline{T Y}\right) \rightarrow 0$ uniformly on compact subsets of $V$. Now the facts that $\overline{T Y} \subset Y$ and that $T$ is bounded imply that $\operatorname{dist}\left((\lambda-T) f_{n}(\lambda), Y\right)$ and $\operatorname{dist}\left(T f_{n}(\lambda), \overline{T Y}\right)$ both converge to zero on compact subsets of $V$. The rest of the proof is similar to that of Theorem 2.8.

The next theorem has analogues for various other types of invariant subspaces - spectral maximal, and analytically invariant [8] subspaces, for example.

We first need a technical lemma; we omit its elementary proof.

2.10. Lemma. Let $X$ be a Banach space, and let $Y$ and $Z$ be subspaces such that $Y \subset Z \subset X$. Let $[x]$ denote the coset in $X / Y$ containing $x$. Then $\operatorname{dist}\left(\left[x_{n}\right], Z / Y\right) \rightarrow 0$ if and only if $\operatorname{dist}\left(x_{n}, Z\right) \rightarrow 0$. 
2.11. TheOREM. Let $T$ be a bounded linear operator on a Banach space $X$, and suppose that $Y$ and $Z$ are $T$-invariant subspaces such that $Y \subset Z \subset X$.

(1) The subspace $Z$ is strongly analytic for $T$ if and only if $Z / Y$ is strongly analytic for $T^{Y}$.

(2) If the subspace $Y$ is T-strongly analytic, then it is also $(T \mid Z)$-strongly analytic.

Proof. The proof of (2) is elementary; we shall prove only (1). Suppose that $f_{n}: V \rightarrow X / Y, n=1,2, \ldots$, are $(X / Y)$-valued analytic functions, that $Z$ is strongly analytic for $T$, and that

$$
\operatorname{dist}\left(\left(\lambda-T^{Y}\right) f_{n}(\lambda), Z / Y\right) \rightarrow 0
$$

uniformly on compact subsets of $V$. Let $K \subset V$ be compact. We may suppose that $K$ is a closed disk; then there exists an open set $U$ in $V$ such that $K \subset U \subset V$, and just as in the proof of Theorem 2.1, we may find $X$-valued analytic functions $F_{n}: U \rightarrow X$, such that $\left[F_{n}(\lambda)\right]=$ $f_{n}(\lambda), n=1,2, \ldots$, where $[x]$ is the coset in $X / Y$ containing $x$. Therefore $\operatorname{dist}\left(\left[(\lambda-T) F_{n}(\lambda)\right], Z / Y\right) \rightarrow 0$ uniformly for $\lambda$ in $K$, and by the lemma, $\operatorname{dist}\left((\lambda-T) F_{n}(\lambda), Z\right) \rightarrow 0$ uniformly for $\lambda$ in $K$. Since $Z$ is $T$-strongly analytic, we have that $\operatorname{dist}\left(F_{n}(\lambda), Z\right) \rightarrow 0$ uniformly for $\lambda$ in $K$, and so $\operatorname{dist}\left(f_{n}(\lambda), Z / Y\right) \rightarrow 0$ uniformly for $\lambda$ in $K$, again by the lemma. This shows that $Z / Y$ is $T^{Y}$-strongly analytic.

For the reverse implication, suppose that $f_{n}: V \rightarrow X, n=1,2, \ldots$, are $X$-valued analytic functions, that $Z / Y$ is $T^{Y}$-strongly analytic, and that $\operatorname{dist}\left((\lambda-T) f_{n}(\lambda), Z\right) \rightarrow 0$ uniformly on compact subsets of $V$. Then $\operatorname{dist}\left(\left(\lambda-T^{Y}\right)\left[f_{n}(\lambda)\right], Z / Y\right) \rightarrow 0$ uniformly on compact subsets of $V$. From the hypotheses, we infer that $\operatorname{dist}\left(\left[f_{n}(\lambda)\right], Z / Y\right) \rightarrow 0$ uniformly on compact subsets of $V$. Another application of the lemma completes the proof.

We turn now to the study of the stability of strongly analytic subspaces under a change of operator.

If $T$ is a bounded linear operator on a Banach space, and $f: U \rightarrow C$ is a scalar-valued analytic function defined on an open set $U$ containing the spectrum of $T$, then by $f(T)$ we mean, as usual,

$$
f(T):=\frac{1}{2 \pi i} \int_{\Gamma} f(\lambda)(\lambda-T)^{-1} d \lambda
$$

where $\Gamma$ is an appropriately chosen curve; see [4; VII.3] for details. 
2.12. Definition [1]. We say that a decomposable operator $T$ is strongly decomposable if the operator $T \mid Y$ is decomposable for every $T$-spectral maximal subspace $Y$.

2.13. TheOREM. Let $T$ be a strongly decomposable operator, and let $Y$ be a spectral maximal subspace for $T$. If $f$ is a scalar-valued analytic function defined on a neighborhood of $\sigma(T)$, then the subspace $Y$ is strongly analytic for $f(T)$.

Proof. By [8], $Y$ is analytically invariant for $T$, and therefore, by [5; Theorems 2.3 and 2.8], $f(T)^{Y}=f\left(T^{Y}\right)$. By [1], $T^{Y}$ is decomposable, and therefore $[3 ; 1.10]$ so is $f\left(T^{Y}\right)$. Thus $f\left(T^{Y}\right)$ has condition $(\beta)$, and since in the present circumstances $f\left(T^{Y}\right)=f(T)^{Y}$, we see by Theorem 2.1, that $Y$ is strongly analytic for $f(T)$.

2.14. THEOREM. Let $T$ be a strongly decomposable operator, let $Q$ be a quasinilpotent operator that commutes with $T$, let $S$ be a finite rank operator that commutes with $T$, and let $Y$ be $T$-invariant.

(1) If $Y$ is $Q$-invariant, then it is strongly analytic for $T+Q$.

(2) If $Y$ is $S$-invariant, then it is strongly analytic for $T+S$.

Proof. We prove (1); the proof of (2) is similar. It is easy to see that $Q^{Y}$ is quasinilpotent on $X / Y$, and so, [3; 2.2.1], $(T+Q)^{Y}=T^{Y}+Q^{Y}$ is decomposable. Thus $(T+Q)^{Y}$ has condition $(\beta)$, and the result follows from Theorem 2.1.

The next theorem is an easy consequence of Lemma 1.6 and the facts that the compact operators form a two-sided ideal, and that for commuting operators, $\sigma(S T) \subset \sigma(S) \sigma(T)$.

2.15. Theorem. Let $T$ be a bounded linear operator, let $Q$ be a quasinilpotent operator that commutes with $T$, and let $K$ be a compact operator. If a subspace $Y$ is invariant under any of the operators, TQ,TK, or $K T$, then it is also strongly analytic for those same operators.

3. Strongly decomposable operators on a reflexive Banach space. In this section we turn to a study of strongly decomposable operators on a reflexive Banach space. The next theorem, a characterization of such operators, is the main theorem of this section. Recall that $Y^{\perp}$ is the orthogonal complement of $Y$ in $X^{*}$, and that $Y^{\perp}$ is $T^{*}$-invariant if $Y$ is $T$-invariant. 
3.1. THEOREM. Let $T$ be a bounded linear operator on a reflexive Banach space. Then $T$ is strongly decomposable if and only if

(1) $T$ has condition $(\beta)$, and

(2) For every spectral maximal subspace $Y$ of $T$, the subspace $Y^{\perp}$ is $T^{*}$-strongly analytic.

Proof. Suppose $T$ is strongly decomposable; then $T$ has condition $(\beta)$. Let $Y$ be a spectral maximal subspace of $T$. Then $(T \mid Y)$ and $(T \mid Y)^{*}$ are decomposable, and so by Lemma $1.6,\left(T^{*}\right)^{Y^{\perp}} \cong(T \mid Y)^{*}$ has condition $(\beta)$. But now by Theorem 2.1, $Y^{\perp}$ is $T^{*}$-strongly analytic.

On the other hand, suppose that $T$ has condition $(\beta)$, and let $Y$ be a spectral maximal subspace for $T$. By Lemma 1.6, the restriction operator $T \mid Y$ has condition $(\beta)$. By hypothesis, $Y^{\perp}$ is strongly analytic for $T^{*}$, so by Theorem 2.1 and Lemma 1.6, $(T \mid Y)^{*} \cong\left(T^{*}\right)^{Y^{\perp}}$ has condition $(\beta)$. Since both $T \mid Y$ and $(T \mid Y)^{*}$ have condition $(\beta), T \mid Y$ is decomposable by Lange's Theorem.

We remark that the first part of the proof did not use the reflexivity of the underlying Banach space. Thus a necessary condition for an operator to be strongly decomposable is that the orthogonal complement of each of its spectral maximal subspaces be strongly analytic for $T^{*}$.

The next result is really a corollary of the proof of Theorem 3.1.

3.2. Corollary. Let $T$ be a decomposable operator on a reflexive Banach space $X$, and let $Y$ be a T-invariant subspace such that $Y^{\perp}$ reduces $T^{*}$. Then $T \mid Y$ is a decomposable operator.

In particular, if the orthogonal complement of each T-spectral maximal subspace reduces $T^{*}$, then $T$ is strongly decomposable.

Proof. Since $T$ is decomposable, so is $T^{*}$, and thus $T^{*}$ has condition $(\beta)$. By hypothesis there exists a $T^{*}$-invariant subspace $Z$ such that $X^{*}=Y^{\perp} \oplus Z$. By Lemma 1.6, the operator $T^{*} \mid Z$ has condition $(\beta)$. Thus $\left(T^{*}\right)^{Y^{\perp}} \cong T^{*} \mid Z$ has condition $(\beta)$ so that $Y^{\perp}$ is $T^{*}$-strongly analytic. Now just as in Theorem 3.1, $(T \mid Y)$ and $(T \mid Y)^{*}$ have condition $(\beta)$, so that $T \mid Y$ is decomposable.

From this last corollary, we can easily obtain a special case of a known result.

3.3. Corollary. Let $T$ be a decomposable operator on a reflexive Banach space $X$. If a subspace $Y$ reduces $T$, then $T \mid Y$ is decomposable. 
Proof. By hypothesis, there exists a $T$-invariant subspace $Z$ such that $X=Y \oplus Z$. Thus we have

$$
X^{*}=Y^{*} \oplus Z^{*} \cong(X / Z)^{*} \oplus(X / Y)^{*} \cong Z^{\perp} \oplus Y^{\perp} .
$$

Hence $Y^{\perp}$ reduces $X^{*}$, and the result follows from Corollary 3.2.

The following example is of interest due to Theorem 3.1.

3.4. EXAMPLE. This is an example of a $T$-strongly analytic subspace $Y$ such that $Y^{\perp}$ is not $T^{*}$-strongly analytic. Let $T$ be the left bilateral shift on $l_{2}(Z)$, and let $Y=\overline{\operatorname{sp}}\left\{e_{-1}, e_{-2}, \ldots\right\}$, so that $Y^{\perp}=\overline{\operatorname{sp}}\left\{e_{0}, e_{1}, e_{2}, \ldots\right\}$ where $\left\{\ldots, e_{-1}, e_{0}, e_{1}, \ldots\right\}$ is the usual orthonormal basis.

Now $\left(T^{*}\right)^{Y^{\perp}}$ is isometric to $(T \mid Y)^{*}$, the left unilateral shift on $l_{2}(N)$ which does not have condition $(\beta)$; for instance, let $f_{n}(\lambda):=\sum_{j=0}^{\infty} e_{j} \lambda^{j}$ on $V:=\{\lambda \in C:|\lambda|<1\}$. Thus by Theorem 2.1, $Y^{\perp}$ is not $T^{*}$-strongly analytic. On the other hand, the operator $T^{Y}$ is isometric to $(T \mid Y)^{* *}$, the right unilateral shift on $l_{2}(N)$, which has condition $(\beta)$ since it is the restriction of the right bilateral shift. Thus by Theorem 2.1, the subspace $Y$ is $T$-strongly analytic.

3.5. REMARK. Example 3.4 shows more than that the orthogonal complement of a strongly analytic subspace need not be strongly analytic. Since the operator $T$ in Example 3.4 is unitary, and therefore spectral, it follows from [4; XV.3.10] that $T$ is strongly decomposable. Thus we see that the necessary condition (2) of Theorem 3.1 may fail for invariant subspaces of a strongly decomposable operator that are not spectral maximal.

Acknowledgement. The author is grateful to the referee for his helpful suggestions, especially those concerning Theorems 2.7 and 2.14, and for pointing out to the author the substance of Remark 3.5.

\section{REFERENCES}

[1] C. Apostol, Restrictions and quotients of decomposable operators in a Banach space, Rev. Roumaine Math. Pures Appl., 13 (1968), 147-150.

[2] E. Bishop, A duality theorem for arbitrary operators, Pacific J. Math., 9 (1959), 379-397.

[3] I. Colojoara and C. Foiaş, Theory of Generalized Spectral Operators, Gordon and Breach, New York, 1968.

[4] N. Dunford and J. T. Schwartz, Linear Operators, Parts I, III, Wiley-Interscience, New York, 1958, 1971.

[5] I. Erdelyi and R. Lange, Spectral Decompositions on Banach Spaces, Lecture Notes in Mathematics \#623, Springer-Verlag, New York, 1977. 
[6] C. Foiaş, On the maximal spectral spaces of a decomposable operator, Rev. Roumaine Math. Pures Appl., 15 (1970), 1599-1606.

[7] Spectral maximal spaces and decomposable operators in Banach space, Arch. Math., (Basel) 14 (1963), 341-349.

[8] S. Frunză, The single-valued extension property for coinduced operators, Rev. Roumaine Math. Pures Appl., 18 (1973), 1061-1065.

[9] R. Lange, A purely analytic criterion for a decomposable operator, Glasgow Math. J., 21 (1980), 69-70.

[10] Strongly analytic subspaces, in Operator theory and functional analysis, edited by I. Erdelyi, Research Notes in Mathematics \#38, Pitman, San Francisco, 1979.

[11] J. C. Snader, Bishop's condition beta and decomposable operators, Ph.D. Thesis, University of Illinois, Urbana, 1982.

Received January 24, 1983 and in revised form April 4, 1983. This material constitutes part of the author's Ph.D. thesis written under the direction of Professor R. G. Bartle at the University of Illinois.

UNIVERSITY OF SOUTH FLORIDA

TAMPA, FL 33620 


\title{
PACIFIC JOURNAL OF MATHEMATICS \\ EDITORS
}

\author{
DoNALD BABBITT (Managing Editor) \\ University of California \\ Los Angeles, CA 90024 \\ J. DugunduI \\ University of Southern California \\ Los Angeles, CA 90089-1113 \\ R. FINN \\ Stanford University \\ Stanford, CA 94305 \\ HERMANN FlasChKa \\ University of Arizona \\ Tucson, AZ 85721
}

C. C. Moore

University of California

Berkeley, CA 94720

ARTHUR OGUS

University of California

Berkeley, CA 94720

Hugo Rossi

University of Utah

Salt Lake City, UT 84112

H. SAMELSON

Stanford University

Stanford, CA 94305

ASSOCIATE EDITORS
R. ARENS
E. F. BECKENBACH
B. H. NeUMANN
F. WOLF
K. YoSHIDA (1906-1982)

\section{SUPPORTING INSTITUTIONS}

UNIVERSITY OF ARIZONA
UNIVERSITY OF BRITISH COLUMBIA
CALIFORNIA INSTITUTE OF TECHNOLOGY
UNIVERSITY OF CALIFORNIA
MONTANA STATE UNIVERSITY
UNIVERSITY OF NEVADA, RENO
NEW MEXICO STATE UNIVERSITY
OREGON STATE UNIVERSITY
UNIVERSITY OF ARIZONA
CALIFORNIA INSTITUTE OF TECHNOLOGY
UNIVERSITY OF CALIFORNIA
UNIVERSITY OF NEVADA, RENO
OREGON STATE UNIVERSITY
UNIVERSITY OF OREGON
UNIVERSITY OF SOUTHERN CALIFORNIA
STANFORD UNIVERSITY
UNIVERSITY OF HAWAII
UNIVERSITY OF TOKYO
UNIVERSITY OF UTAH
WASHINGTON STATE UNIVERSITY
UNIVERSITY OF WASHINGTON 


\section{Pacific Journal of Mathematics}

Vol. 115, No. $1 \quad$ September, 1984

Carlos Andradas Heranz and José Manuel Gamboa Mutuberría, A note on projections of real algebraic varieties $\ldots \ldots \ldots \ldots \ldots \ldots \ldots \ldots \ldots \ldots$

Jürgen Appell and Maria Patrizia Pera, Noncompactness principles in nonlinear operator approximation theory $\ldots \ldots \ldots \ldots \ldots \ldots \ldots \ldots \ldots \ldots$

Timothy John Carlson, Extending Lebesgue measure by infinitely many

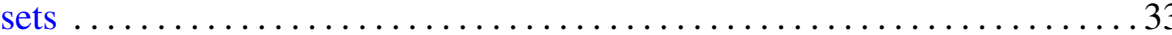

Donald S. Coram and Paul Frazier Duvall, Jr., Non-cell-like

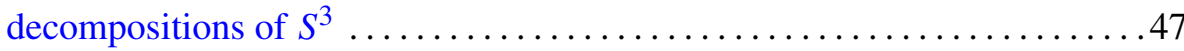

Edward Norman Dancer, Order intervals of selfadjoint linear operators and

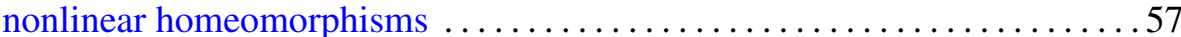

Ralph Jay De Laubenfels, Well-behaved derivations on $C[0,1] \ldots \ldots \ldots 73$

D. Feyel and A. de La Pradelle, Sur certaines extensions du théorème

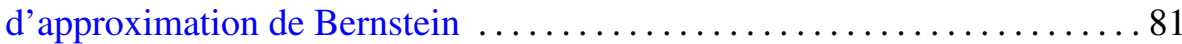

Colin C. Graham and Bertram Manuel Schreiber, Bimeasure algebras on

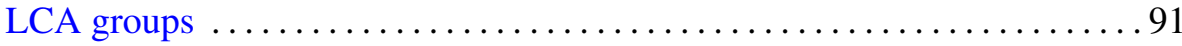

Richard Howard Hudson, Class numbers of imaginary cyclic quartic fields

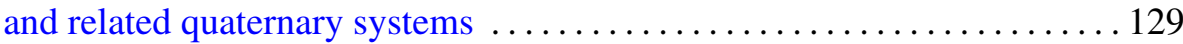

Carl Groos Jockusch, Jr. and Iraj Kalantari, Recursively enumerable sets and van der Waerden's theorem on arithmetic progressions . . . . . . . . 143

J. F. McClendon, On noncontractible valued multifunctions ........... 155 Akihiko Miyachi, Weak factorization of distributions in $H^{p}$ spaces $\ldots \ldots \ldots 165$ Ezzat S. Noussair and Charles Andrew Swanson, Global positive solutions of semilinear elliptic problems

Jon Christopher Snader, Strongly analytic subspaces and strongly

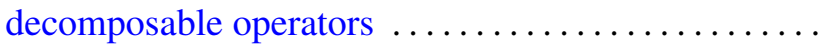

Boguslaw Tomaszewski, A construction of inner maps preserving the Haar measure on spheres

Akihito Uchiyama, The Fefferman-Stein decomposition of smooth functions and its application to $H^{p}\left(\mathbf{R}^{n}\right) \ldots \ldots \ldots$ 